\title{
Impacto del aprendizaje activo de la Microbiología en el Grado de Podología
}

\author{
Impact of active learning: Teaching Microbiology in the degree of Podiatry
}

\author{
María Luisa Gómez-Lus, Carmen Ramos, Luis Alou, José Prieto \\ Facultad de Medicina. Area de Microbiología (Departamento de Medicina). Universidad Complutense de Madrid. \\ mlgomezl@ucm.es; carmenramos@ucm.es; luisalou@ucm.es; jprieto@ucm.es
}

Correspondencia:

María Luisa Gómez-Lus Centelles

Area de Microbiología

Departamento de Medicina

Facultad de Medicina

Avda. Complutense s/n

28040 Madrid

Correo electrónico: mlgomezl@ucm.es

Fecha de recepción: 8 de junio de 2014

Fecha de aceptación: 2 de octubre de 2014

Los autores declaran no tener ningún tipo de interés económico o comercial.

\section{RESUMEN}

Con la adaptación al Espacio Europeo de Educación Superior, se ha planteado cómo objetivo la mejora de la calidad de la docencia de la Microbiología mediante la renovación de la metodología docente, introduciendo como actividad docente el aprendizaje activo basado en preguntas (inquiry-based learning:IBL) para conseguir mejorar las competencias que deberán adquirir los estudiantes como parte de su formación integral. En este estudio han participado 49 alumnos de Segundo Curso del Grado de Podología,y se ha calculado el porcentaje de alumnos que participaron en las 3 pruebas (3 IBL), en 2 (2 IBL), en 1 (1 IBL), y los que no participaron en ninguna y posteriormente se relacionó con las calificaciones obtenidas en la asignatura de Microbiología. En los alumnos que realizaron alguna prueba IBL se obtuvieron calificaciones mejores en las preguntas diseñadas para analizar la síntesis de conocimientos y el análisis de datos que en aquellos que no habían participado en ninguna. Al finalizar la actividad se realizó un estudio transversal a través de un cuestionario autocumplimentado en el que se valoraba la opinión de los alumnos sobre el aprendizaje activo mediante IBL valorando positivamente esta actividad para medir el aprendizaje y mejorar la preparación del examen. Consideramos que en líneas generales este tipo de estudios pueden optimizar la calidad de la docencia de la Microbiología en los estudios de Grado de Podología.

Palabras clave: Microbiología; Podología; aprendizaje activo; calidad; docencia.

\begin{abstract}
In response to the Adaptation to the European Higher Education Area, one path to reform is the development of educational practices that explicit engage students while enhacing how they learn Microbiology. These practices including inquiry-based learning (IBL) in which students are actively engaged in the learning process for improving the skills to be acquired as part of their effective learning. This study included 49 Microbiology students of Second Year Degree in Podiatry, and calculated the percentage of students who used these resources 3 IBL, 2IBL, 1IBL, and that did not participate in any and subsequently related to the student performance in Microbiology exam. Students who prepared IBL scored higher en exam questions designed to test synthesis of knowledge and analysis of data. In order to assess the perceived impact of the active learning, the students were asked to fill out a questionnaire evaluating IBL positively considering that this activity can measure learning and improve exam preparation. We believe that this type of study can optimize the quality of teaching of Microbiology in podiatric studies.
\end{abstract}

Keywords: Microbiology; Podiatry; active learning; quality; teaching.

Sumario: 1. Introducción. 2. Material y Métodos. 3. Resultados. 4. Discusión. Bibliografía.

Referencia normalizada: Gómez-Lus, M.L., Ramos, C., Alou, L., Prieto J. Impacto del aprendizaje de la Microbiología en el Grado de Podología. Rev. Int. Cienc. Podol. 2015; 9(1): 17-23. 


\section{INTRODUCCIÓN}

La Universidad Complutense de Madrid fue una de las primeras Universidades españolas que aprobó los estudios de Podología para la obtención del Título de Diplomado en Podología impartiéndose la asignatura de Microbiología en el primer curso. Con la adaptación al Grado, se ha comenzado a impartir Microbiología dentro de la materia Biopatología en segundo curso $^{1}$. Se ha planteado cómo objetivo la mejora de la calidad de la docencia mediante la renovación de la metodología docente cómo en otras Universidades donde la actualización de los objetivos docentes es constante $\mathrm{e}^{2,3,4}$.

Los alumnos de Microbiología tienen un tiempo limitado para estudiar conceptos complejos que permitan asentar las bases para el diagnóstico de las enfermedades infecciosas en la Podología. Dentro de los cambios realizados para la adaptación al espacio de educación europea (EEES) y crear entornos de aprendizaje reflexivo en materias científicas que establezcan conexiones entre el aprendizaje teórico y la aplicación práctica profesional se plantea como un reto la renovación de la metodología docente.

El aprendizaje basado en preguntas (inquirybased learning: IBL) es una metodología centrada en el autoaprendizaje guiado por el profesor, en la reflexión que siguen los alumnos para responder a las preguntas planteadas en la explicación de cada tema, permitiendo un pensamiento crítico, tomando parte activa en el aprendizaje que además permite el trabajo cooperativo mediante la interrelación con los compañeros. El IBL se plantea como un medio diagnóstico para valorar errores conceptuales antes de comenzar la clase y que sea un punto de partida para la adquisición en integración de nuevos conocimientos. Mediante esta metodología los alumnos actúan cómo parte activa del aprendizaje $^{5,6}$. Los objetivos del aprendizaje se han definido siguiendo las directrices de la Sociedad Americana de Microbiología (ASM) en la que plantea objetivos sencillos (Lower-Order thinking) y complejos (Higher-order thinking) según la Taxonomía de Bloom ${ }^{7,8}$. Por otra parte se ha comprobado cómo este tipo de aprendizaje incrementa el rendimiento académico mejorando las calificaciones de la asignatura y la valoración de la asignatura ${ }^{9,10}$.Tambien y cómo ya se ha realizado previamente en los estudiantes de Medicina ${ }^{11}$ y en los de Podología ${ }^{1}$, se ha consultado la valoración y las opiniones que tienen los alumnos de segundo de Podología sobre el proceso de enseñanza-aprendizaje de la Microbiología.

\section{MATERIAL Y MÉTODOS}

El estudio se realizó por 49 alumnos que cursaban Biopatología durante el Curso 2013-2014 y que se imparte en $2^{\circ}$ de Grado de la titulación de Podología de la Universidad Complutense de Madrid.

Al inicio de la exposición de un grupo de 3 temas se realizaba una prueba compuesta por varias preguntas a las que podían contestar con verdadero o falso, al finalizar esa unidad temática, contestaban de nuevo esas preguntas y valoraban los aciertos conseguido antes de la clase y después de la clase. Se realizaron 3 tandas de IBL, el primero correspondió a 12 preguntas de la parte de Microbiología general (IBL-A), La segunda a 15 preguntas la parte de Bacteriología (IBL-B) y la tercera a 15 preguntas la parte de Parasitología (IBL-C).

Se ha calculado el porcentaje de alumnos que participaron en las 3 pruebas (3 IBL), en 2 (2 IBL), en 1 (1 IBL), y los que no participaron en ninguna y posteriormente se relacionó con las calificaciones obtenidas en la asignatura de Microbiología.

Posteriomente se desglosó el contenido del examen, que contaba de 35 preguntas, de las que 27 median conceptos básicos desarrollados en la clase teórica y 8 integraban conceptos más complejos ampliados mediante IBL, en la teoría vs. teoría con IBL y la nota obtenida.

Para comparar el porcentaje de aciertos previo a la clase (preclases) y posterior a la clase (postclases) en los alumnos que entregaron las tres pruebas (IBL A, B y C) se empleó el test exacto de Fisher.

Al finalizar actividad se realizó un estudio transversal a través de un cuestionario autocumplimentado en el que se valoraba la opinión de los alumnos sobre el aprendizaje reflexivo mediante IBL recibida, resaltando aspectos para mejorar las competencias y 
habilidades alcanzadas con esta metodología docente. Esta encuesta fue cumplimentada por 25 alumnos.

La participación en todas las pruebas era voluntaria y no se tenía en cuenta para la calificación final.

\section{RESULTADOS}

Del total de alumnos, 12 (24,48\%) alumnos participaron y entregaron corregidos las tres tandas de IBL (3 IBL), 12 (24,48\%) alumnos lo hicieron con dos tandas de IBL (2 IBL) y 16 alumnos $(32,65 \%)$ entregaron solamente una (1 IBL), mientras que 9 alumnos $(18,36 \%)$ no entregaron ningún IBL (Figura 1).

En relación a las calificaciones de la asignatura, de los alumnos que realizaron 3 IBL, 3 (25\%) obtuvieron sobresaliente, 7 (58,3\%) notable, y $2(16,6 \%)$ aprobado, destacando que en este grupo no hubo ningún suspenso, Del grupo que realizaron 2 IBL, $3(25 \%)$ obtuvieron sobresaliente, $4(33,3 \%)$ notable, y $4(33,3 \%)$ aprobado y suspenso $1(8,3 \%)$. Del grupo que realizó 1 IBL, $3(18,75 \%)$ obtuvo sobresaliente, $8(50 \%)$ notable, y $3(18,75 \%)$ aprobado y suspenso $2(12,5 \%)$.

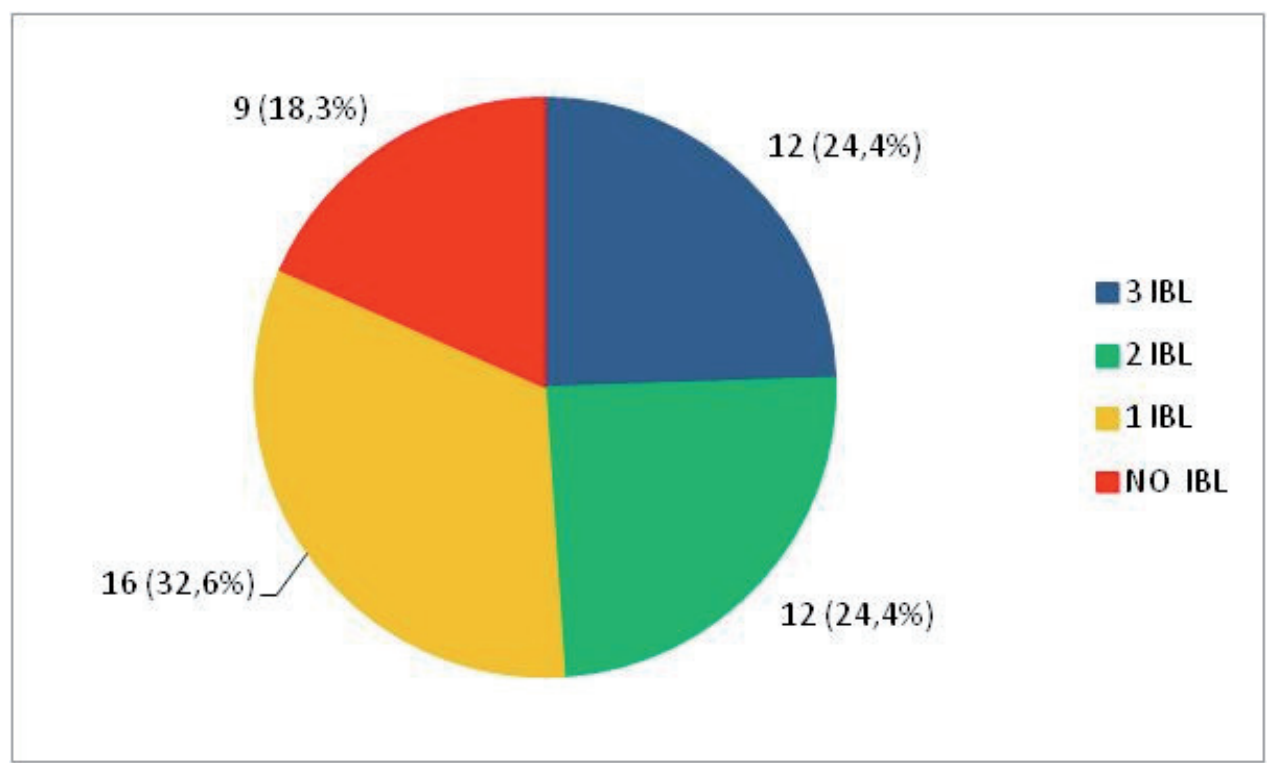

Figura 1. Número de alumnos (\%) que realizaron las pruebas IBL.

\begin{tabular}{lrrrrr} 
& $\begin{array}{r}\text { Total } \\
\mathbf{n}(\mathbf{\%})\end{array}$ & $\begin{array}{r}\mathbf{3} \text { IBL } \\
\mathbf{n}(\mathbf{\%})\end{array}$ & $\begin{array}{r}\text { 2 IBL } \\
\mathbf{n}(\mathbf{\%})\end{array}$ & $\begin{array}{r}\mathbf{1} \text { IBL } \\
\mathbf{n}(\mathbf{\%})\end{array}$ & $\begin{array}{r}\text { No IBL } \\
\mathbf{n}(\mathbf{\%})\end{array}$ \\
\hline Sobresaliente & $10(20,40)$ & $3(25,0)$ & $3(25,0)$ & $3(18,75)$ & $1(11,1)$ \\
Notable & $22(44,89)$ & $7(58,3)$ & $4(33,3)$ & $8(50)$ & $3(33,3)$ \\
Aprobado & $12(24,48)$ & $2(16,6)$ & $4(33,3)$ & $3(18,75)$ & $3(33,3)$ \\
Suspenso & $5(10,20)$ & $0(0,0)$ & $1(8,3)$ & $2(12,5)$ & $2(22,2)$ \\
\hline
\end{tabular}

Tabla 1. Calificaciones agrupadas por la entrega de los diferentes IBL, alumnos que entregaron 3 IBL, 2 IBL, 1 IBL y los que no entregaron ninguno (No IBL). 


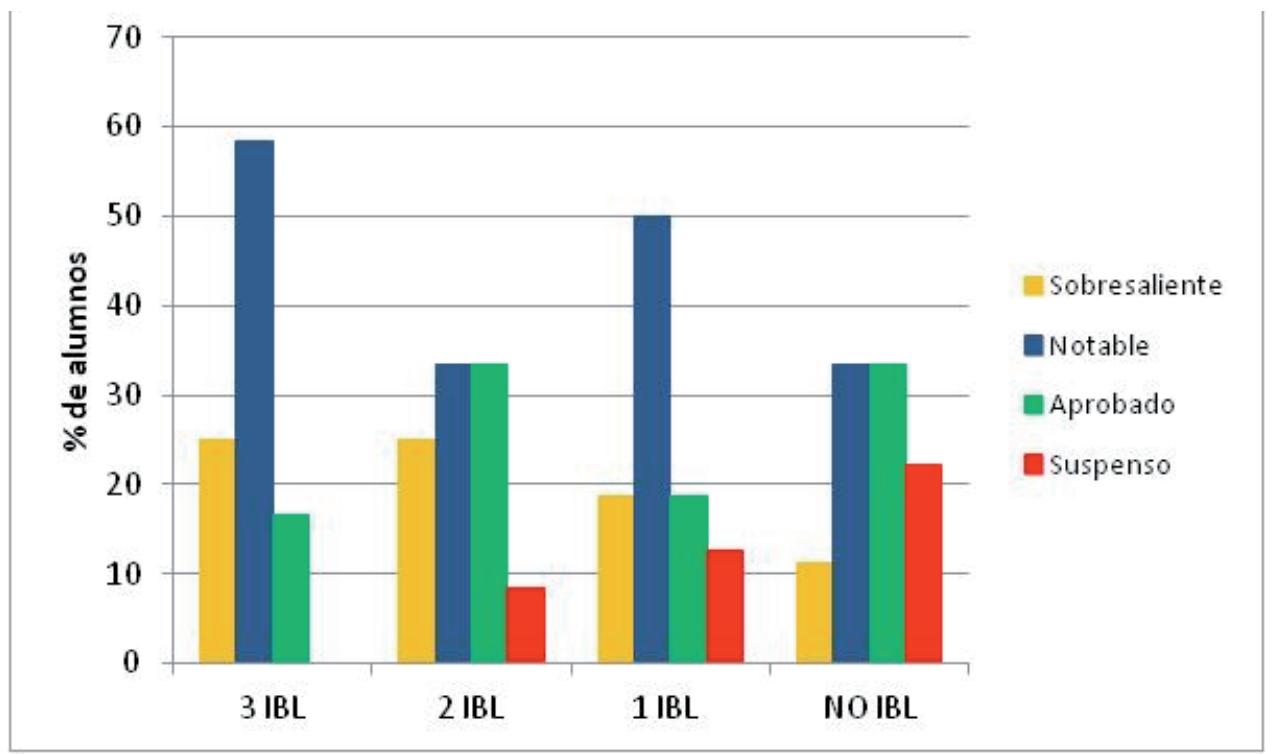

Figura 2. Calificaciónes agrupadas por la entrega de los diferentes IBL alumnos que entregaron 3 IBL, 2 IBL, 1 IBL y los que no entregaron ninguno (No IBL).

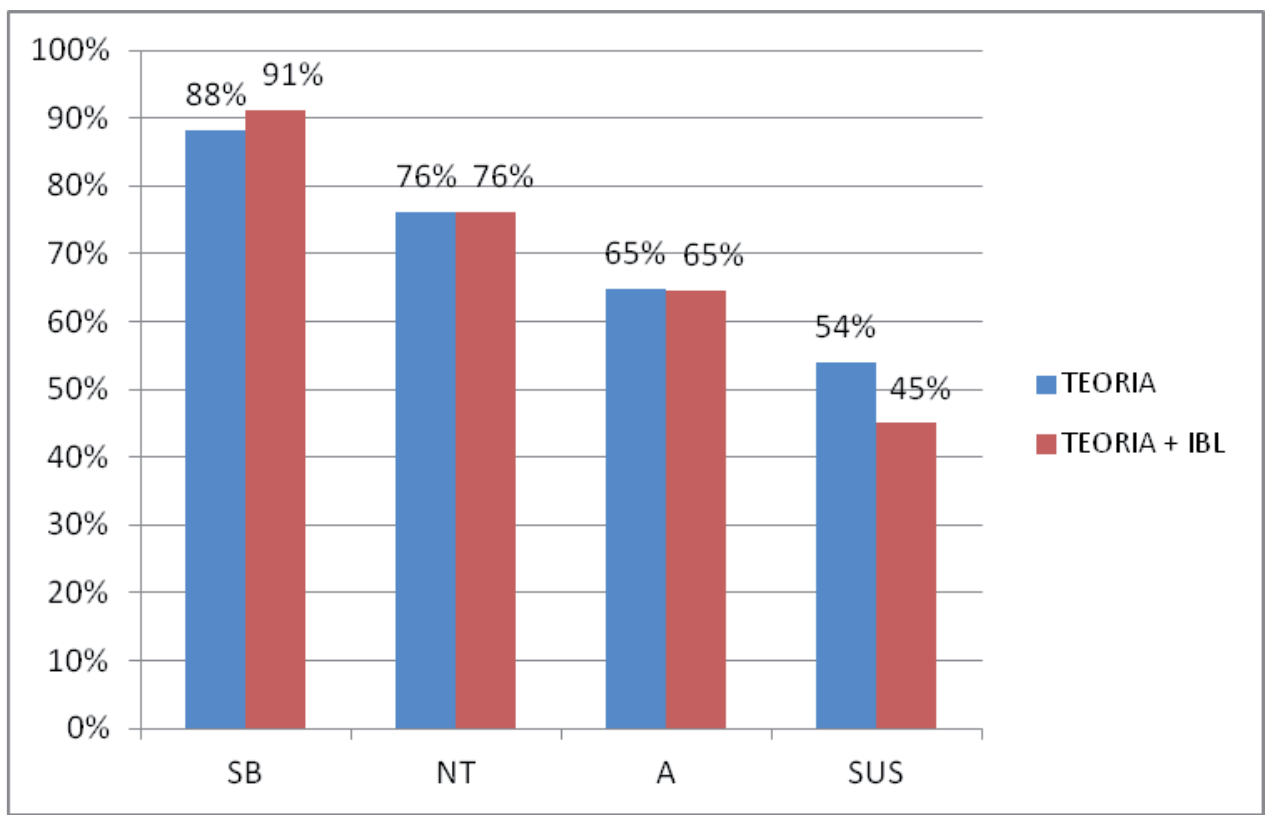

Figura 3. Porcentaje de aciertos relacionado con el tipo de aprendizaje: teoría de menor complejidad (teoria) o teoria con IBL de mayor compejidad (teoria con IBL) en la superación de la asignatura. 
Del grupo sin IBL, 1 (11,1\%) obtuvo sobresaliente, $3(33,3 \%)$ notable, y $3(33,3 \%)$ aprobado y suspenso 2 (22,2\%) (Figura 2).

Dentro del examen de la asignatura, se estructuro en una parte de conceptos más sencillos que explicaron mediante explicación teórica (Lower-order thinking skills) a y otra parte de conceptos más complejos (Higher-order thinking skills) que se habían reforzado con el método IBL, al estudiar el índice de aciertos, comprobando cómo pese a ser conceptos mas complicados, los resultados eran similares e incluso mejores en el grupo de los alumnos que obtuvieron sobresaliente (Figura 3).

En relación a los porcentajes de aciertos de las diferentes preguntas IBL, se analizó el incremento del aprendizaje en los alumnos que entregaron las tres pruebas IBL, 12 alumnos. Se compararon los porcentajes de aciertos al inicio de la clase (pretest) y al finalizar la clase (postest).

En el primer bloque de IBL (IBL A), en el test previo se obtuvo un $66 \%$ y en el posterior un $83 \%$; en el segundo bloque de IBL (IBL B) en el test previo se alcanzó un 59\% y en el posterior un $87 \%$, mientras que en el tercer bloque de IBL (IBL C) en el test previo se incrementó hasta un $67 \%$ y en el posterior un $97 \%$ (Figura 4). Se observaron diferencias significativas $(p<0,0001)$ entre el porcentaje de aciertos obtenido antes de la clase (preclase) y después de la clase (postclase) para todos los IBL (A, B y C).

Al finalizar las clases se valoró el aprendizaje reflexivo basado en preguntas por parte de los alumnos (Tabla 2), obteniendo la mejor valoración en la pregunta número 6 , ya que esta actividad les había permitido una mejor preparación del examen de la asignatura y la pregunta número 8 ya que medían el aprendizaje en cada una de las unidades temáticas. Por otra parte en la pregunta numero 7 consideraban que sería útil el incluir este tipo de hojas de trabajo en todos los temas de la asignatura. En relación a los comentarios menos favorables fueron la número 5 , ya que sólo un $44 \%$ de los alumnos consideraba que les sería útil para su futura práctica profesional y la número 4 en la que un $32 \%$ refería que con este tipo de ejercicios no había aumentado su interés por la Microbiología.

\section{En relación a las preguntas abiertas todos los alumnos}

informaron que habían mejorado el conocimiento sobre los temas planteados incrementando su atención en clase y que hubieran necesitado más tiempo para rellenar las hojas de trabajo.

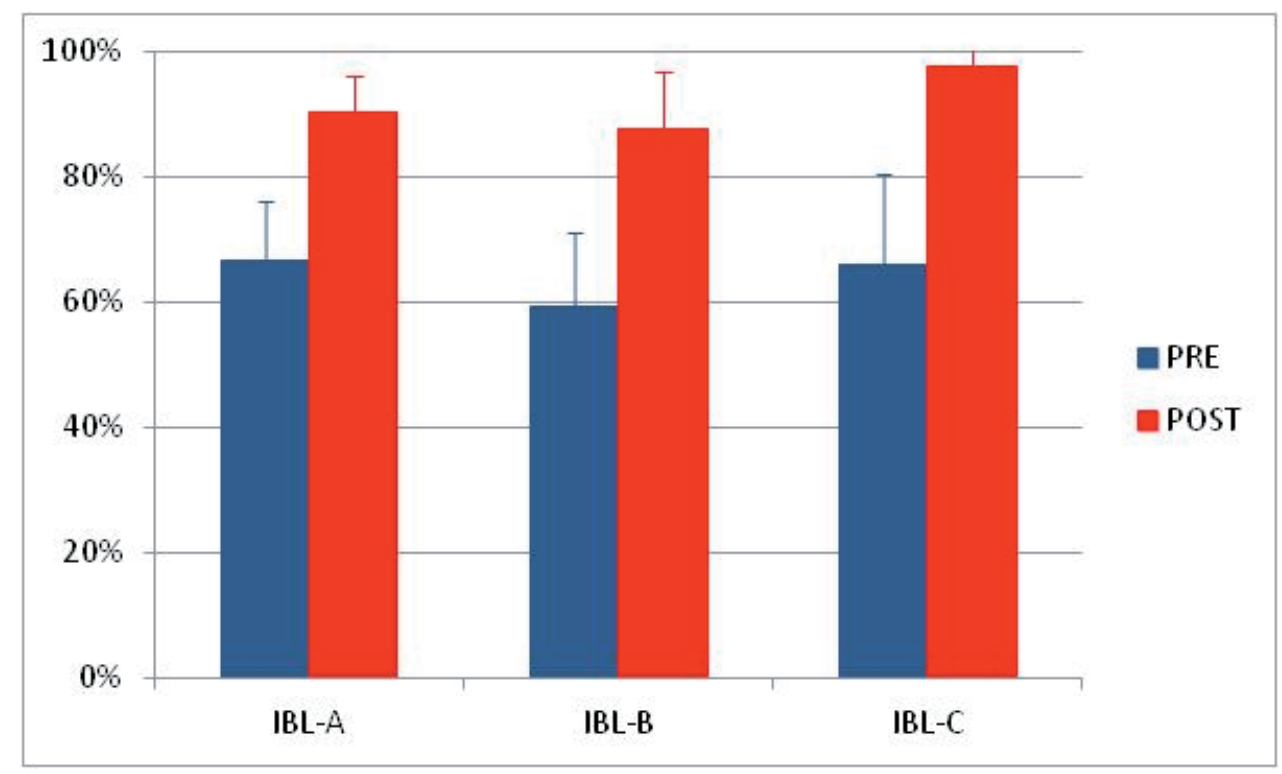

Figura 4. Porcentaje de aciertos antes de la clase (PRE) y despues de la clase (POST) en el primer bloque de IBL (IBL-A), en el segundo bloque de IBL (IBL-B) y en el tercer bloque de IBL (IBL-B). 


\begin{tabular}{|c|c|c|c|c|c|}
\hline & $\begin{array}{r}1 \\
\text { n }(\%)\end{array}$ & $\begin{array}{r}2 \\
\text { n }(\%)\end{array}$ & $\begin{array}{r}3 \\
\text { n }(\%)\end{array}$ & $\begin{array}{r}4 \\
n(\%)\end{array}$ & $\begin{array}{r}5 \\
\text { n }(\%)\end{array}$ \\
\hline 1. Ha mejorado mi aprendizaje sobre el tema. & & & $4(6)$ & $16(64)$ & $5(20)$ \\
\hline 2. La actividad es clara. & & $1(4)$ & $5(20)$ & $9(36)$ & $10(40)$ \\
\hline 3. He consolidado más mis conocimientos. & & $1(4)$ & $5(20)$ & $14(56)$ & $5(20)$ \\
\hline $\begin{array}{l}\text { 4. Ha aumentado mi interés por la Microbio- } \\
\text { logía. }\end{array}$ & & $1(4)$ & $7(28)$ & $12(48)$ & $4(16)$ \\
\hline $\begin{array}{l}\text { 5. La actividad me ha permitido reflexionar } \\
\text { con mis compañeros sobre algún aspecto útil } \\
\text { en mi futura práctica profesional. }\end{array}$ & & $3(12)$ & $11(44)$ & $10(40)$ & $1(4)$ \\
\hline $\begin{array}{l}\text { 6. Esta actividad me ha permitido preparar- } \\
\text { me mejor el examen de la asignatura de Mi- } \\
\text { crobiología. }\end{array}$ & & & $1(4)$ & $15(60)$ & $9(36)$ \\
\hline $\begin{array}{l}\text { 7. Esta actividad se incluiría en todos los te- } \\
\text { mas de la asignatura. }\end{array}$ & & & $4(16)$ & $11(44)$ & $10(40)$ \\
\hline $\begin{array}{l}\text { 8. Mediante esta actividad puedo medir mi } \\
\text { mejora en el aprendizaje. }\end{array}$ & & & $2(12)$ & $12(48)$ & $11(44)$ \\
\hline $\begin{array}{l}\text { 9. Esta actividad me ha permitido detectar } \\
\text { errores conceptuales. }\end{array}$ & & & $4(16)$ & $13(52)$ & $8(32)$ \\
\hline
\end{tabular}

1. Fuertemente desacuerdo; 2. Moderadamente desacuerdo; 3. Indiferente; 4. Moderadamente de acuerdo; 5. Fuertemente de acuerdo.

Tabla 2. Valoración de los estudiantes de Podología 2013-2014 sobre el aprendizaje reflexivo basado en preguntas.

\section{DISCUSIÓN}

El porcentaje de alumnos que no participó fue de un $18.36 \%$, lo que ha incrementado notablemente el interés por parte de los alumnos ya que en trabajos publicados previamente con alumnos de Microbiología General de la Facultad de Medicina de la Universidad Complutense de Madrid el grado de no participación era de un $51 \%{ }^{5}$. Al comparar los resultados académicos de los alumnos se observa cómo los alumnos que realizaron voluntariamente las tres pruebas IBL superaron todos la asignatura de Microbiología. En trabajos previos ya demostramos cómo las actividades prácticas en la docencia de la Microbiología mejoraron el rendimiento académico de los alumnos disminuyendo el número de suspensos ${ }^{1}$. En los últimos años se ha comprobado cómo la implementación de méto- dos de aprendizaje activo han mejorado la calidad de la educación obteniendo mejor rendimiento académico que con la lección magistral en la que el alumno escuchaba y tomaba notas mientras que en los métodos de aprendizaje activo el alumno procesa y aplica la información respondiendo preguntas, solucionando y discutiendo problemas y completando hojas de trabajo $^{12,13}$

Así, la utilización de esta metodología puede contribuir a mejorar el aprendizaje activo y considerarse cómo una estrategia más dentro del proceso de enseñanza y aprendizaje, incentivando el estudio y puede fomentar el aprendizaje cooperativo.

Al ser una prueba voluntaria, destaca la participación inconstante de los alumnos que no entregan las hojas de trabajo corregidas pero que posiblemente utilicen cómo herramienta 
de aprendizaje autónomo al no considerar los resultados de esta prueba necesarios para la calificación.

La participación en la valoración de este aprendizaje reflexivo fue de casi un $50 \%$, mientras que en el trabajo presentado en el Congreso de Docencia y Difusión de la Microbiología 2012 que analizaba la valoración de las clases prácticas en el curso 2011-2012 en los alumnos de Microbiología de Podología fue de un $100 \%$ al ser una prueba obligatoria. Pero estas observaciones, en su mayoría positivas, son interesantes ya que en futuros trabajos nos plantearemos cómo objetivo incrementar los puntos peor valorados, adecuando las preguntas planteadas a su futuro desarrollo profesional cómo Podólogos que permita aumentar su motivación estableciendo una conexión entre la ciencia que se estudia en las aulas y la que se aplica en una clínica podológica.

Consideramos que en líneas generales este tipo de estudios pueden optimizar la calidad de la docencia de la Microbiología en los estudios de Grado, así ya la vista de los resultados obtenidos en los siguientes cursos se reducirán el número de clases magistrales y se incrementarán las prácticas, seminarios y otros procesos de autoaprendizaje, aspectos potenciados en el EEES.

\section{BIBLIOGRAFÍA}

1. Gómez-Lus ML, Alou L, Ramos MC, Prieto J, Adaptación al EEES de la enseñanza de la Microbiología en el Grado de Podología, Congreso del grupo de Docencia y Difusión de la Microbiología de la Sociedad Española de Microbiología, Madrid 12 y 13 de Julio de 2012.

2. Johnson MT. Impact of on line learning modules on medical student Microbiology examination scores. 2008. J. Microbiol. Biol. Educ. 2008; 9: 25.29.

3. Lee VS and Hyman M. Helping educational reforms to succeed in a Microbiology Department. Microbe. 2009; 4. 5: 219-223.

4. Chaudhry MA. Assessment of Microbiology students' progress with an audience response system, J. Microbiol. Biol. Educ. 2011; 12.2: 200-201.

5. Gómez-Lus ML, Alou L, Ramos MC, Prieto J, Aplicación de nuevas metodologías en el aprendizaje de la Microbiología, XI Congreso Nacional de la SEQ, Madrid, 5-8 Octubre 2011.

6. Rodriguez-Avial C.. Gómez-Lus ML. Picazo J. Aplicación del aprendizaje guiado por preguntas a la docencia de Microbiología Médica. Congreso del grupo de Docencia y Difusión de la Microbiología de la Sociedad Española de Microbiología. Madrid 12 y 13 de Julio de 2012.

7. Working Document: ASM GENERAL MICROBIOLOGY LEARNING OUTCOMES. http://www. asm.org/images/Education/Working_Final_ASMGenMicro_LO_-_v_Feb_4_2014.pdf (Ultimo acceso: 9 julio 2014).

8. ASM Recommended Curriculum Guidelines for Undergraduate Microbiology Education Background http://www.asm.org/images/final_curriculum_guidelines_\%20w._intro_4.12.20-b.pdf (Ultimo acceso: 9 julio 2014).

9. Peteroy-Kelly MA. A discussion group enhances the conceptual reasoning skills of students enrolled in a large lecture-format introductory biology course. J. Microbiol. Biol. Educ.2007; 8: 13-21.

10. Peteroy-Kelly MA. Online pre-laboratory modules enhance introductory biology students' preparedness and performance in the laboratory. J. Microbiol. Biol. Educ. 2010; 11: 5-13.

11. Mirón-Canelo JA. Valoración de los estudiantes sobre su formación en la Facultad de Medicina. Educ. Med. 2011; 4: 221-228.

12. Freeman S. et al. Active learning increases student performance in science, engineering, and mathematics. Proc. Nat. Acad. Sci. USA 2014; 111: 8410-8415.

13. Wieman C E. Large-scale comparison of science teaching methods sends clear message. Proc. Nat. Acad. Sci USA 2014; 111: 8319-8320. 